J. Lake Sci. (湖泊科学), 2014, 26(4):625-631

http://www. jlakes. org. E-mail : jlakes@niglas.ac.cn

(c) 2014 by Journal of Lake Sciences

\title{
湖泊沉积物不同粒级组分有机碳同位素差异及其对实验结果的影响”
}

\author{
王君兰 ${ }^{1}$, 邓 伟 $^{2}$, 张平宇 $^{3}$, 张家武 $^{3}$ \\ (1:中国科学院兰州文献情报中心,兰州 730000) \\ ( 2 :甘肃省地质调查院,兰州 730000$)$ \\ ( 3 : 兰州大学西部环境教育部重点实验室,西部环境与气候变化研究院,兰州 730000)
}

\begin{abstract}
摘 要: 湖泊沉积物有机质碳同位素因常被用于识别沉积物中有机质来源或流域植被信息而逐渐成为一个常规代用指 标,但当沉积物中有机质含量变化显著、赋存状态不同时,采用统一分析方法 (全样或某一粒级组分) 测试的结果在不同 含量或赋存状态时是否会产生偏差, 目前没有详细的研究进行评价. 利用嘎顺诺尔湖泊沉积物, 采用全样品、细颗粒组分 (过 120 目篮和 360 目篮), 分别进行有机质含量和同位素分析,评价选择不同粒级样品因有机质赋存状态不同对有机碳 同位素分析结果的影响. 结果显示, 不同组分的烧失量或元素分析仪方法测得的有机质含量变化趋势相同,但不同组分 的有机碳同位素结果出现差异: 全样的有机碳同位素值存在较大波动, 随着过篮孔径变小, $\delta^{13} \mathrm{C}_{\mathrm{org}}$ 值波动减小, 且过 360 目篮的细颗粒组分的碳同位素值较全样或过 120 目篮后组分的 $\delta^{13} \mathrm{C}_{\mathrm{org}}$ 值偏负. 这一差异与有机质组分赋存的颗粒范围有 关. 对比认为过 360 目篮的细颗粒组分更有利于充分反应, 且可获得较为准确的同位素值. 因此,在进行不同湖泊沉积物 $\delta^{13} \mathrm{C}_{\mathrm{org}}$ 值对比时, 应注意研究使用样品的前处理方式, 相同处理方式下的结果更具有可比性. 该研究结果对于湖泊沉积物 有机质碳同位素分析具有参考意义.
\end{abstract}

关键词: 湖泊沉积物; 全样品; 细颗粒组分; 有机碳同位素

\section{The differences in organic carbon isotope of different size components in lake sediments and its impact on experimental results}

\author{
WANG Junlan ${ }^{1}$, DENG Wei ${ }^{2}$, ZHANG Pingyu ${ }^{3}$ \& ZHANG Jiawu ${ }^{3}$ \\ (1: Lanzhou Library of Chinese Academy of Sciences, Lanzhou 730000, P. R. China) \\ (2: Gansu Institute of Geological Survey, Lanzhou 730000, P. R. China) \\ (3: MOE Key Laboratory of Western China's Environmental Systems/Research School of Arid Environment and Climate \\ Change, Lanzhou University, Lanzhou 730000 , P. R. China)
}

\begin{abstract}
The organic carbon isotope (OCI) of lake sediment has often been used to identify the source of organic matter in sediments or watershed vegetation, and gradually becomes a routine proxy indicator. However, when organic matter content changes significantly and in different forms, the OCI results from the unified approach (bulk sample or a graded composition) will produce deviation, no studies have evaluated this problem so far. This research chose sediment samples from rump lake in arid areas, analyzed the organic content and isotope using bulk sample and fine-grained components ( screened through mesh size of 120 and 360 ), respectively. The influence on OCI results based on different sediment grain sizes and with different organic matter forms was evaluated. Results reveal different OCI values in those sediment even with similar organic matter from different sediment components. Obviously, this relates to the forms of organic matter. It shows that fine-grained components ( screened through mesh size of 360 ) is more advantageous to full reaction, and obtain a more stable value. When comparing the $\delta^{13} \mathrm{C}_{\text {org }}$ value in different lake sediments, researchers should adapt same methods for sample pretreatment. The result has an important implication for the research on organic carbon isotope in lake sediments.
\end{abstract}

Keywords: Lake sediments; bulk sample; fine-grained components; $\delta^{13} \mathrm{C}_{\text {org }}$

* 国家重点基础研究发展计划“973”项目 (2009CB421306) 和国家自然科学基金项目 (40871093,41021091) 联合资 助. 2013-06-04 收稿;2013-07-22 收修改稿. 王君兰(1985 ), 女, 硕士, 助理馆员; E-mail: wangjl@ llas. ac. cn. 
湖泊沉积物中的有机质含量是反映湖泊或流域生产力和湖泊沉积环境的常规指标, 其碳同位素 $\left(\delta^{13} \mathrm{C}_{\text {org }}\right)$ 可以反映湖泊或流域内植被类型的变化, 从而反映气候环境的变化. 因此有机质含量及其 $\delta^{13} \mathrm{C}_{\text {org }}$ 常 被用来恢复区域的古环境、古植被变化情况 ${ }^{[1-7]}$. 常见的有机质含量的测定方法有烧失法 ( LOI)、滴定法、元 素分析仪测定法 (TOC) 等, 前人的研究结果表明, 尽管不同方法测定的值存在差异, 但变化趋势相同 ${ }^{[8-13]}$, 均 能代表沉积物中有机质含量的变化情况. 碳同位素 $\delta^{13} \mathrm{C}_{\text {org }}$ 的测定一般选择全岩样品, 并需要去除样品中较大 的植物残体及无机碳酸盐, 避免由此带来样品的不均一性, 同时较好地保留有机质组分, 才能使实验结果较 准确地反映古气候环境的变化 ${ }^{[14-20]}$. 但对于有机质含量变化较大的湖泊沉积物, 如出现较多植物残体时, 目 视挑选去除植物残体 (全样不过篮, 研磨足够细且均匀即可) 和篎除掉植物残体的细颗粒组分 (如过 120 目 篮和 360 目篮等) 对样品均一性的影响、其同位素组成的差别以及对于古环境解释的影响尚无详细研究和 评估. 本文选取内蒙古额济纳旗黑河下游嘎顺诺尔 GXN 剖面的样品, 通过选用不同大小颗粒组分的样品进 行有机质含量及有机碳同位素的对比实验, 讨论不同组分有机质碳同位素结果的差异, 为湖泊沉积物有机 质碳同位素分析提供优选方案.

\section{1 材料与方法}

\section{1 实验材料}

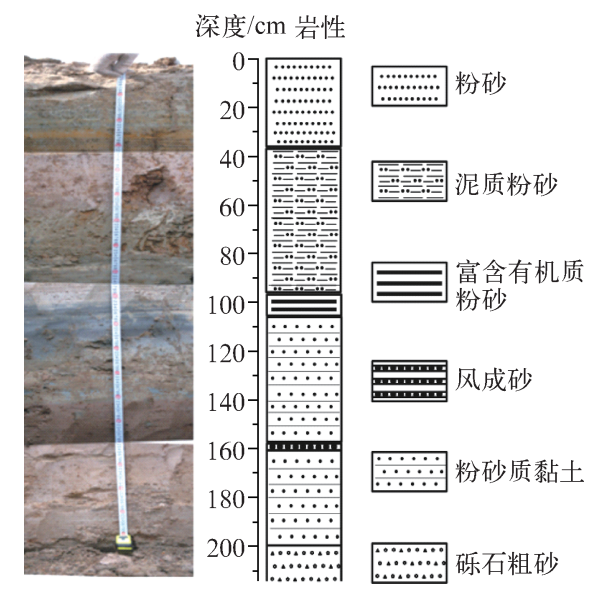

图 1 嘎顺诺尔 GXN 剖面图及其岩性

Fig. 1 Photo of the GXN section and lithological profile
本文采用的沉积物样品于 2009 年 11 月在嘎顺诺尔东 部 $\left(42^{\circ} 27^{\prime} 03^{\prime \prime} \mathrm{N}, 100^{\circ} 54^{\prime} 05^{\prime \prime} \mathrm{E}\right)$ 采集, 海拔 $890 \mathrm{~m}$. 四周是干涸 的湖底, 在湖底上挖掘探坑剖面 (GXN) 至地下水出露为止, 探坑深 $2 \mathrm{~m}$ (图 1). 在剖面上以 $1 \mathrm{~cm}$ 间隔采样, 共采集样品 200 个, 样品装人自封袋密封后运回室内, 经真空冷冻干燥 仪干燥后待分析.

\section{2 实验方法}

1.2. 1 烧失量法 将坩埚用稀盐酸清洗后放在预先烧至 $550^{\circ} \mathrm{C}$ 的马弗炉中灼烧 $4 \sim 6 \mathrm{~h}$, 恒重后称重, 称约 $1 \mathrm{~g}$ 干样 (精确到 $0.0001 \mathrm{~g}$ ) 放人坩埚中, 将坩埚放人 $105^{\circ} \mathrm{C}$ 烘箱干燥 $12 \mathrm{~h}$, 称重后在马弗炉中 $550^{\circ} \mathrm{C}$ 灼烧 $2 \sim 3 \mathrm{~h}$, 关闭电源, 打开 炉门, 待部分热量散失后, 用长钳取出坩埚置于干燥器中冷 却. 待温度降至室温时在分析天平上称重. 烧失部分的重量 占样品干重的百分比即为烧失量 $(L O I)^{[21]}$. 样品的有机质 含量计算公式为: $L O I(\%)=\left(550^{\circ} \mathrm{C}\right.$ 时烧失量 $/$ 干重 $) \times 100 \%$.

1.2.2 有机质含量及有机碳同位素的测定 1) 将经冷冻干 燥过的样品分为 3 份, 一份挑取掉对土壤有机质有影响的大的植物残体, 未经过篮直接将全样用玛瑙研钵 研磨均匀; 另一份样品挑取掉植物残体后用蒸馏水分层湿篮, 上层篮孔孔径为 120 目 $(125 \mu \mathrm{m})$, 下层孔径为 360 目 $(45 \mu \mathrm{m})$, 将通过下层 $45 \mu \mathrm{m}$ 孔径的细粒部分烘干后研磨均匀; 另外挑选含植物残体较多的样品 (91 115 cm) 过 120 目篮, 烘干后用玛瑙研针研磨均匀;

2) 透水坩埚置于马弗炉中 $550^{\circ} \mathrm{C}$ 灼烧 $4 \mathrm{~h}$ 左右, 取出置于干燥器中冷却;

3) 取上述适量样品 (参照烧失量结果视有机质的多少而定) 放在透水坩埚中, 将透水坩埚置于培养血 中, 用 $10 \%$ 的盐酸滴人透水坩埚中, 滴加至反应缓和时, 加人过量的盐酸充分反应 $24 \mathrm{~h}$, 目的是去除样品中 的碳酸盐;

4) 将透水坩埚放在能将其支起并透水的架子上, 在透水坩埚中加人蒸馏水洗至中性 (用 $\mathrm{pH}$ 试纸测 试), 将透水坩埚放在烘箱中, $75^{\circ} \mathrm{C}$ 烘 $24 \mathrm{~h}$, 将充分干燥后的样品混合均匀, 用玛瑙研针研磨至 120 目左右, 装人小试管中编号保存备用;

5) 用万分之一天平称取样品, 并以干净的特制小锡舟紧密包好 (包样过程尽量将锡舟中空气排挤干 净, 否则空气中的 $\mathrm{CO}_{2}$ 会影响到样品测定值的准确性), 然后放人旋转式自动载样盘中进行上机 (元素分析 
仪一同位素比例质谱仪-EA-IRMS) 测试, 误差 $<0.2 \%$. 对于每个样品, 必须予以准确称量, 燃烧释放的 $\mathrm{CO}_{2}$ 在 通过色谱柱后与标准样品的 $\mathrm{CO}_{2}$ 气体的峰积分面积相比较, 从而得到待测样品的碳含量 ( 以元素 $\mathrm{C}$ 的质量 百分数形式给出), 即总有机碳 (TOC) ${ }^{[22]}$.

以上实验均在兰州大学西部环境教育部重点实验室完成.

\section{2 结果与讨论}

\section{1 烧失法及元素分析仪法测得的总有机碳含量的对比}

准确测定沉积物中总有机碳含量, 需要有效地将有机碳和无机碳区分开来. 烧失法采用特定温度下 $\left(550^{\circ} \mathrm{C}\right)$ 的烧失量来表示有机质含量; 研究表明, 高镁碳酸盐在 $400^{\circ} \mathrm{C}$ 以下会发生分解,而一些难降解的有机 物要在 $1050^{\circ} \mathrm{C}$ 以上才能被氧化,所以该方法在测量样品时产生误差较大,且不易控制. 而元素分析仪法采用 无氧化性的盐酸溶液溶解掉沉积物中的无机碳酸盐, 然后上机测试有机碳含量. 实验过程中也会造成偏差, 比如去除碳酸盐过程可能将可溶性有机物溶解,但相对而言更易控制 ${ }^{[13]}$.

从图 2 可以看出,代表有机质含量的 3 条曲线 $(a, b$ 和 $c$ ) 形态相似,说明不同组分不同分析方法测得的 有机质含量变化趋势一致. 整个剖面有机碳含量较低,仅在 $85 \sim 115 \mathrm{~cm}$ 段有机质含量较高, 与野外相应位置 观察到该段有大量芦苇的植物残体相一致. 对太湖现代沉积物的研究 ${ }^{[23]}$ 表明, 在生有芦苇等水生植物的水 域有机质含量高, 烧失量也较高; 在敞水区或湖心区, 有机质含量低, 烧失量亦低, 烧失量与有机质含量之间 存在密切的关系. 剖面中植物残体含量高段烧失量大致为全样有机碳含量的 2 倍, 这与 Dean 等 $^{[9]}$ 的研究结 果一致.

全样样品在 $85 \sim 115 \mathrm{~cm}$ (植物残体含量高) 段的 TOC(图 2b) 较细颗粒组分的 TOC(图 2a) 含量高, 其余 层位全样比细颗粒组分有机质含量稍低. 这表明在有机质含量较低的湖泊沉积物中,不溶解的有机质颗粒 主要为较细的颗粒, 过篮去除了比重较大的沉积物颗粒后, 该部分有机质所占的比例相应增加. 而含植物残 体多的湖泊沉积物中, 过篮过程中多数粗颗粒有机质被篎除,过篎后的细颗粒组分 TOC 含量较全样低. 尽管 实际研究中烧失量因测定起来快速简便、经济的特点而经常用来代表样品有机质含量,但因前处理不同 (未 去除碳酸盐), 在有机质含量较低的沉积物中, 烧失量波动较大 (图 2c), 可能与不同样品碳酸盐含量、可溶 有机组分不同以及有机质烧失过程中部分碳酸盐分解有关. 因此用烧失量来粗略代表有机质含量时, LOI 曲 线与其他指标对比时,其细节变化不具有对比意义,这在研究中应引起注意.

烧失法和元素分析仪法测得的 TOC 含量的相关性分析 (图 3) 表明, 同一样品 (如全样, 图 3a)TOC(元素 分析) 和 $L O I$ 尽管统计结果相关 $(n=200)$, 但部分数据点仍较为离散. 过篮后的 TOC 与全样的 LOI(图 $3 \mathrm{~b}$ ) 相关性最低,这与上述有无前处理过程有关;元素分析仪测得的全样和过篮的细颗粒组分的 TOC 相关性最 好 (图 3c), 在有机质含量低时, 二者较为接近, 有机质含量高时, 二者数据也较为离散, 说明取不同组分进行 元素分析测量时,样品的均一性对测量结果影响较大. 过篮的细颗粒组分在有机质含量低时段出现一定的 波动 (图 2a) , 而在有机质含量高的时段波动反而较小. 这反映了过篮过程对有机质的分选作用: 细颗粒组分 相对富集,粗颗粒的有机质(如残体)得到有效去除.

\section{2 全样和细颗粒组分有机碳同位素 $\delta^{13} \mathrm{C}_{\mathrm{org}}$ 值的对比}

嘎顺诺尔 GXN 剖面沉积物全样和细颗粒组分的 $\delta^{13} \mathrm{C}_{\text {org }}$ 值曲线形态相似 (图 $2 \mathrm{~d} 、 \mathrm{e}$ ), 细颗粒组分有机 碳同位素变化范围为 $-25.51 \% 0 \sim-19.23 \%$, 平均值为 $-23.03 \%$; 全样有机碳同位素值在 $-24.88 \%$ $-18.36 \%$ 之间, 平均值为 $-22.63 \%$. 全样比细颗粒组分的有机碳同位素值偏正, 两者的差值 (D-value, 图 2f) 变化范围为 $-1.51 \sim 3.13$, 平均值为 0.39 . 两者之间的差值也可能与有机质组分赋存 的颗粒范围有关. 为了进一步分析不同粒径的样品测得的碳同位素值结果, 选取植物残体含量较多的 $91 \sim 115 \mathrm{~cm}$ 段样品, 增加过 120 目篎组分的样品进行测试, 并与全样和过 360 目篎的细颗粒组分进行 对比, 结果如图 4 .

同一样品不同粒径组分的有机碳 $\delta^{13} \mathrm{C}_{\text {org }}$ 值趋势基本一致 (图 4), 但全样的 $\delta^{13} \mathrm{C}_{\text {org }}$ 值 (图 4a) 波动最大, 过 120 目篎组分的 $\delta^{13} \mathrm{C}_{\text {org }}$ 值 (图 4c) 次之, 过 360 目篎组分的 $\delta^{13} \mathrm{C}_{\text {org }}$ 值 (图 4b) 波动最小. 对同一样品不同组分 的 $\delta^{13} \mathrm{C}_{\text {org }}$ 值进行相关分析, 结果如图 5. 全样的 $\delta^{13} \mathrm{C}_{\text {org }}$ 值与过不同孔径篮子组分的 $\delta^{13} \mathrm{C}_{\text {org }}$ 值相关程度均不高 




图 2 嘎顺诺尔 $\mathrm{GXN}$ 剖面全样和细颗粒组分的有机质含量及 $\delta^{13} \mathrm{C}_{\mathrm{org}}$ 值曲线

Fig. 2 TOC content and $\delta^{13} \mathrm{C}_{\text {org }}$ of the bulk sample and the fine-grained components of the GXN section
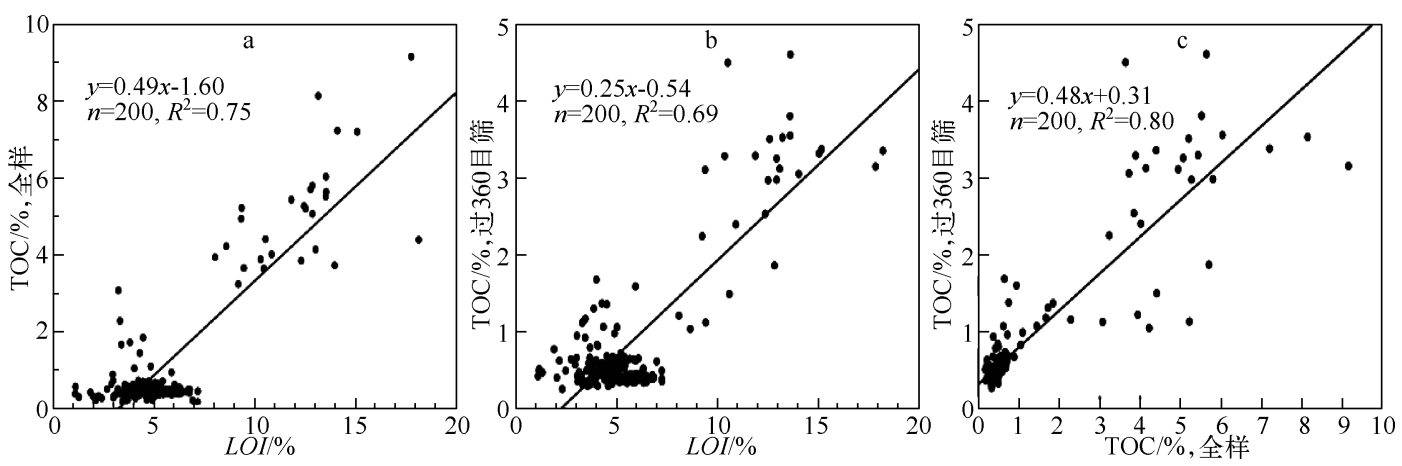

图 3 烧失法和元素分析仪法测得有机质含量结果的相关关系

Fig. 3 Relationship of organic contents by LOI and elemental analysis methods

(图 5a、b), 但过 120 目篮和 360 目耖后的沉积物 $\delta^{13} \mathrm{C}_{\mathrm{org}}$ 值相关性十分显著 $\left(R^{2}=0.94\right.$ ) (图 5c), 证明过篮的 过程对有机质进行了分选, 使样品更加均匀, 同时分选出的有机质颗粒可能更集中于某一相同来源的有机 质 (如陆源的 $\mathrm{C}_{3}$ 植物), 使过 360 目篎的最细颗粒的 $\delta^{13} \mathrm{C}_{\text {org }}$ 值偏负一些. 颗粒粒径对固体样品的均匀混合也 会产生一定影响. 一般而言, 颗粒粒径越小, 样品越容易混合均匀, 相应的分析结果离散度越小 ${ }^{[24]}$. 如图 5 显 
示全样比细颗粒组分的离散度要大. 因此 本文的实验结果表明, 随着湖泊沉积物全 样被不同孔径篮分后, 有机质含量及其 $\delta^{13}$ $\mathrm{C}_{\text {org }}$ 值均发生变化, 随着过篮的孔径变细, 有机质中的细颗粒碎屑相对富集, 残体影 响减小, 同位素结果波动减小. 因此使用湖 泊沉积物有机质 $\delta^{13} \mathrm{C}_{\text {org }}$ 值进行区域对比 时, 应查明所使用样品的处理方式, 相同处 理方式下的 $\delta^{13} \mathrm{C}_{\text {org }}$ 结果更具有可比性. 波 动较大的 $\delta^{13} \mathrm{C}_{\text {org }}$ 结果可能是样品不均匀所 致. 对比结果表明, 过 360 目篮后的样品, 细颗粒有机质相对富集, 样品混合更均匀, 有利于燃烧更为充分, 反应更加完全, 更有 利于分析区域有机质 $\delta^{13} \mathrm{C}_{\mathrm{org}}$ 的稳定变化趋 势, 从而排除剧烈波动给指标对比和区域 对比带来的干扰. 此外, 嘎顺诺尔 GXN 剖 面沉积物全样和细颗粒组分 (过 360 目篮) 的 TOC 与 $\delta^{13} \mathrm{C}_{\text {org }}$ 值的相关性 (图 6) 均很 低, 说明有机质碳同位素值与有机质含量



图 4 嘎顺诺尔 GXN 剖面沉积物全样 和细颗粒组分的 $\delta^{13} \mathrm{C}_{\text {org }}$ 值对比曲线

Fig. 4 Comparison of the $\delta^{13} \mathrm{C}_{\text {org }}$ of the bulk sample and the fine-grained components of the GXN section 关系不大.


图 5 嘎顺诺尔 GXN 剖面沉积物全样和细颗粒组分的 $\delta^{13} \mathrm{C}_{\mathrm{org}}$ 值的相关关系

Fig. 5 Relationship of $\delta^{13} \mathrm{C}_{\text {org }}$ values between bulk sample and fine-grained components of the GXN section

\section{3 结论}

通过分析嘎顺诺尔湖泊沉积物不同组分有机质含量及其有机碳同位素值,得到以下结论：

1) 湖泊沉积物有机质含量较高时, 烧失量 $L O I$ 大致相当于元素分析仪法得到的全样 TOC 的 2 倍; 有机 质含量较低时, LOI 与 TOC 差别较大, 因为 TOC 分析前处理时去除了可溶的有机质组分; 全样的 TOC 较细 粒 TOC 略低, 显示出过篮过程中对有机质颗粒的分选作用. 两种方法存在很好的相关性, 比较而言, 烧失量 测定方法仅能粗略地用来代表有机质含量, 与其他指标对比时细节变化可能不代表环境意义; 用元素分析 仪测定得到的 TOC 结果更准确.

2) 嘎顺诺尔 GXN 剖面沉积物全样和细颗粒组分的 $\delta^{13} \mathrm{C}_{\text {org }}$ 值对比表明,全样的有机碳同位素值存在较 大波动, 随着过篮孔径变小, $\delta^{13} \mathrm{C}_{\mathrm{org}}$ 值趋于稳定, 表明 $\delta^{13} \mathrm{C}_{\mathrm{org}}$ 值与样品均匀程度有关, 且过 360 目篎的细颗粒 组分的碳同位素值较全样或过 120 目篮后组分的 $\delta^{13} \mathrm{C}_{\mathrm{org}}$ 偏负. 这一差异与有机质组分赋存的颗粒范围有关, 䇻后分选 (富集) 了低有机质含量段的细颗粒有机质,也剔除了大颗粒残体的影响, 因此我们认为过 360 目 篮的细颗粒组分更有利于充分反应, 且可获得更有利于区域对比的同位素值. 不同湖泊沉积物 $\delta^{13} \mathrm{C}_{\mathrm{org}}$ 值对比 

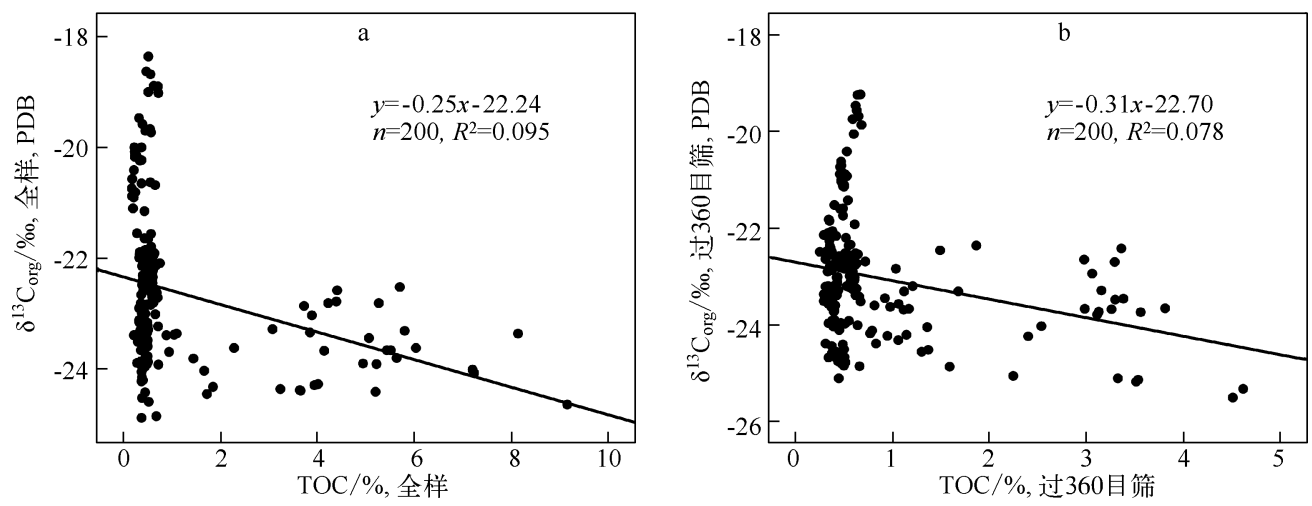

图 6 嘎顺诺尔 GXN 剖面沉积物全样/细颗粒组分的 TOC 与 $\delta^{13} \mathrm{C}_{\mathrm{org}}$ 值的相关关系

Fig. 6 Relationship of TOC and $\delta^{13} \mathrm{C}_{\text {org }}$ value of bulk sample or fine-grained components of the GXN section 时, 应注意研究使用样品的前处理方式是否相同, 相同处理方式下的 $\delta^{13} \mathrm{C}_{\mathrm{org}}$ 值更具有对比意义.

\section{4 参考文献}

[ 1 ] 吴敬禄, 王苏民. 湖泊沉积物中有机质碳同位素特征及其古气候. 海洋地质与第四纪地质, 1996,16(2):103-109.

[ 2 ] 沈 吉, 王苏民, 羊向东. 湖泊沉积物中有机碳稳定同位素测定及其古气候环境意义. 海洋与湖沼, 1996, 27 (4): 400-403.

[3] 沈 吉,吴瑞金,安芷生. 大布苏湖沉积剖面有机碳同位素特征与古环境. 湖泊科学, 1998, 10(3):8-12.

[ 4 ] 余俊清, 王小燕, 李 军等. 湖泊沉积有机碳同位素与环境变化的研究进展. 湖泊科学, 2001, 13(1):72-78.

[ 5 ] 张恩楼, 沈 吉, 夏威岗等. 青海湖沉积物有机碳及其同位素的气候环境信息. 海洋地质与第四纪地质, 2002,22 (2) : 105-108.

[ 6 ] 马 龙, 吴敬禄. 安固里淖湖积物中总有机碳含量及其碳同位素的环境意义. 自然资源学报, 2009, 24 (6): 1099-1104.

[ 7 ] 吴敬禄,王苏民. 湖泊沉积物有机质所揭示的环境气候信息. 湖泊科学, 1996,8 (2):113-118.

[ 8 ] 王浩然,陈业材. 湖泊沉积物有机碳分析的最优化方法. 贵州环保科技, 1996,2(4) :21-24.

[ 9 ] Jr Dean WE. Determination of carbonate and organic matter in calcareous sediments and sedimentary rocks by loss on ignition: Comparison with other methods. Journal of Sedimentary Research, 1974, 44 : 242-248.

[10] Heiri O, Lotter AF, Lemcke G. Loss on ignition as a method for estimating organic and carbonate content in sediments : reproducibility and comparability of results. Journal of Paleolimnology, 2001, 25 : 101-110.

[11] Santisteban JI, Mediavilla R, Lopez-Pamo E et al. Loss on ignition: a qualitative or quantitative method for organic matter and carbonate mineral content in sediments? Journal of Paleolimnology, 2004, 32 : 287-299.

[12] Beaudoin A. A comparison of two methods for estimating the organic matter content of sediments. Journal of Paleolimnology, 2003, 29: 387-390.

[13] 卢凤艳, 安芷生. 鹤庆钻孔沉积物总有机碳、氮含量测定的前处理方法及其环境意义. 地质力学学报, 2010, 16 (4): $393-401$.

[14] 曹蕴宁,刘卫国,宁有丰等.土壤有机碳同位素样品制备过程的影响因素讨论. 地球化学,2005,34(4):395-404.

[15] 王金权. 样品预处理对有机碳同位素分析结果的影响. 古生物学报,2005,44(3):472-477.

[16] 吴 夏,黄俊华,白 晓等. 沉积岩总有机质碳同位素分析的前处理影响. 地球学报,2008,29(6):677-683.

[17] 刘 强,刘嘉麒,陈晓雨等. $18.5 \mathrm{kaB}$. P. 以来东北四海龙湾玛珥湖全岩有机碳同位素记录及其古气候环境意义. 第 四纪研究, 2005,25 (6) : 711-721.

[18] Midwood AJ, Boutton TW. Soil carbonate decomposition by acid has little effect on $\delta^{13} \mathrm{C}$ of organic matter. Soil Biology and Biochemist, 1998, 10 (11) : 1301-1307.

[19] Schubert CJ, Nielsen B. Effects of decarbonation treatments on $\delta^{13} \mathrm{C}$ values in marine sediments. Marine Chemistry, 
$2000,72(1): 55-59$.

[20] Lorrain A, Savoye N, Chauvaud L et al. Decarbonation and preservation method for the analysis of organic C and N contents and stable isotope ratios of low-carbonated suspended particulate material. Analytica Chimica Acta, 2003, 491(2) : 125-133.

[21] 张家武,金 明,陈发虎等. 青海湖沉积岩芯记录的青藏高原东北部过去 800 年以来的降水变化. 科学通报,2004, 49(1) : 10-14.

[22] 饶志国,陈发虎,曹 洁等. 黄土高原西部地区未次冰期和全新世有机碳同位素变化与 $\mathrm{C} 3 / \mathrm{C} 4$ 植被类型转换研究. 第四纪研究,2005,25(1):107-113.

[23] 袁旭音,陈 俊,季俊峰等. 太湖现代沉积物的物质组成和形成条件分析. 南京大学学报: 自然科学版, 2002,38 (6) :756-765.

[24] 喻 涛, 李春园. 盐酸温度时间及粒径对海洋沉积物碳酸盐去除的影响. 热带海洋学报,2006,25(6):33-38. 